\title{
Sur les pontes anormales chez Philine aperta $\mathrm{L}$.
}

Par

\author{
Dr. Jan Tur.
}

(Du Laboratoire Zootomique de l'Université de Varsovie.)

Avec 3 figures dans le texte.

Eingegangen am 28. Februar 1910.

En m'intéressant aux questions de l'origine et de la formation des monstres doubles, j'étudiais à plusieurs reprises, pendant mes séjours successifs au Laboratoire de Zoologie de Roscoff, - les pontes anormales de Philine aperta L. (ancienne Bullaea aperta Lam.) où la fréquence des larves doubles était signalée depuis plus de trente ans par le célèbre Fondateur du laboratoire. C'est HenrI de Lacaze - Duthiers qui a publié les premières observations sur la formation des larves véligères doubles dans les pontes de ce Gastéropode 1 ), observations accompagnées de dessins, qui ont jusqu'ici conservé tonte leur valeur. C'est le travail de LACAzE-Durniers et aussi les observations ultérieures de M. J. GUIaRT ${ }^{2}$ ) qui m'ont servi de point $d u$ départ pour mes recherches. A mon savoir cette intéressante question n'a été depuis soulevée nulle part et e'est pourquoi je me décide à publier mes observations, quoiqu'elles soient encore bien incomplètes.

On sait bien, comment ont échoué toutes les tentatives de provoquer par la voie expérimentale le développement des monstres doubles comparables à ceux qui naissent spontanément

1) H. DE LACAZE-Duthiers: ?Sur la formation des monstres doubles chez les Gastéropodes. Archives de Zoologie Expérimentale et Générale. T. IV. 1875. Pl. XV.

2) Jules Guiart: >Contributions à l'étude des Gastéropodes Opisthobranches et en particulier des Céphalaspides." Paris 1901. 
dans la nature. C'est pourquoi que l'observation de H. DE LACAZE-Duthiens devait depuis longtemps attirer l'attention des tératogénistes, car elle seule aurait permis d'obtenir à son gré et en appliquant les procédés les plus simples - des monstres doubles en quantité suffisante pour leur étude approfondie. D'après H. DE LACAZE-Duthiers en tracassant les Philines pendant qu'elles pondent on les force à activer la sortie des aeufs et alors ceux-ci, au lieu d'être enfermé chacun dans une coque ovigère séparée, - sont déposés par deux ou mềme trois dans une même coque. Ces œufs, d'abord tout à fait indépendants l'un de l'autre, se soudent ensuite et produisent les monstres doubles, dont les types divers ont été figurés par H. De LaCaze-Duthilers et M. J. Guiart.

C'est en 1902 que j'ai commencé mes études sur les pontes anormales de Philine. Dès mes premières observations j'ai constaté, que dans les pontes cueillies sur la plage, et par consequent, pondues dans les conditions normales, on peut trouver des différences notables de la fréquence des coques à deux oufs: dans les pontes, déposées sur la plage de Pempoul (près de Saint-Pol de Léon) ces coques anormales étaient excessivement rares, tandis que dans les pontes provenant de Santec (à l'ouest de Roscoff́) - les coques à deux et même à trois oufs se rencontraient presque constamment et parfois leur quantité surpassait sensiblement celle des coques normales. Quelquefois j'ai vu même des coques, renfermant quatre et même cinq cufs à la fois, ce qui présente un fait nouveau. En étudiant le développement ultérieur de ces embryons évoluant dans la coque commune, je n'ai constaté qu'une quantité insignifiante de larves véligères doubles, tandis que dans l'immense majorité des cas les coques aux œufs multiples fournissaient autant des larves normales, tout à fait indépendantes, sans les moindres traces de soudure réciproque. -

Mes expériences avec le tracassement des Philines pendant la ponte m'ont conduit anx résultats tont à fait différents de ceux qui ont été signalés par oe LaCaze-Duthiers. Les animaux, surpris pendant la ponte et fortement inquiétés aux coups de scalpel ete. - essayaient toujours, en effet, d'activer la sortie des oeufs, le chapelet de ceux-ci paraissait bien plus serré et irrégulier, - mais, tout de même, chaque ceuf se trouvait déposé dans une coque séparée et d'aspect normal. En modifiant l'expérience de telle façon, qu'en tirant sur le bout du filament d'œufs déjà pondus - j'activais directement la ponte - j'obtenais quelquefois l'apparition de quel- 
ques centimètres de fil anormal, contenant de longues tubes ovigères, anormalement retrécies, renfermant deux, trois, jusqu'à sept œufs accolés à la fois. Les oufs pondus dans ces conditions, trop serrés les uns contre les autres, commencaient ordinairement très tard leur segmentation et les embryons y périssaient bientôt.

En 1905 et 1908 j'essayais de répéter mes expériences, mais depuis 1903 les Philines, jusqu'alors assez fréquentes dans les environs de Roseoff ont disparu subitement ou, au moins, sont devenues tellement rares, que, malgré le concours tonjours si aimable de l'Administration du Laboratoire, - je ne pouvais me procurer qu'une quantité insuffisante d'individus adultes et de pontes. C'est en 1909 que ce Mollusque a réappara en abondance, surtont sur la grève de Pempoul - de sorte, que je pouvais reprendre mes observations sur un matériel largement suffisant. Alors, en disposant d'une soixantaine d'individus adultes, qui m'ont fourni pendant six semaines 170 pontes environ, j'ai répété mes observations et expériences d'auparavant, en les élargissant par l'étnde statistique de coques anormales, qui se sont présenté abondamment dans quelques unes de ces pontes.

Les premières pontes étaient, pour la plupart, tout à fait normales. Je les ntilisais pour mes expériences sur l'influence des rayons du radium, dont j'ai déjà publié les résultats (C. R. Acad. Sc. 1909), et en même temps j'en ai profité pour répéter mes expériences avec le tracassement des animaux, surpris pendant la ponte. Les résultats de ces expériences étaient toujours les mêmes: une poute normalement commencée, s'achevait anssi d'une façon normale malgré toutes les lésions mécaniques, auxquelles je soumettais l'animal. Alors, en profitant d'expériences d'électro-physiologie, qui ont été en même temps installées par mon confrère M. J. SosNowski, j’ai essayé à soumettre les Philines pendant la ponte - à l'influence de fortes secousses électriques, produites par la bobine d'induction (Grand modèle de Verdin. Courant $110 \mathrm{~V}$. passait par une lampe à 16 bougies; bobine secondaire à $12 \mathrm{ctm}$.). Dans ces conditions l'animal se tordait littéralement tout en continuant de pondre, mais, si la ponte était normale avant le commencement de l'expérience elle continuait toujours de l'être: les Philines électrisées ne pondaient jamais que des oufs, renfermés dans des coques séparées (Fig. 1). Le fil ovigère sort alors plus vite que d'ordinaire, en s'entortillant d'une façon irrégulière; parfois les œufs se disposent en doubles rangées, mais comme nons le voyons sur la microphotographie ci-jointe, il n'y en a jamais de coques plusiovulaires et même 
chaque coque conserve son diamètre normal. Ajoutons, que parfois, probablement sous l'effet de la contraction des bords de l'orifice génital, - les œufs étaient écrasés, mais je n'ai jamais vu de coques doubles.

Ainsi, en m'appuyant sur les résultats de plus de cinquante expériences, j'arrive à conclure, qu'il est impossible, en tracassant les Philines pendant qu'elles pondent - de provoquer artificiellement l'apparition des pontes anormales a ux coques pluriovulaires: les individus, dont l'appareil génital est normalement constitué, pondent des coques simples, normales,

Fig. 1.

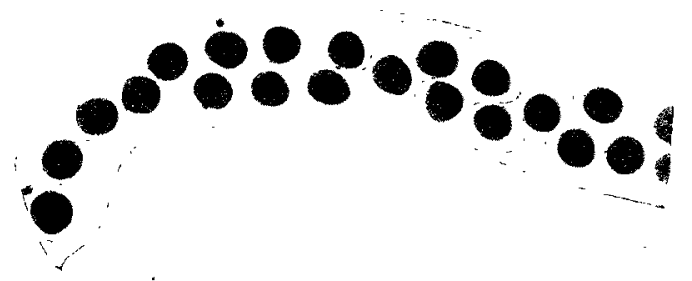

Un fragment du fil ovigère de Philine aperta, pondu pendant l'électrisation. Les aufs sont déposés dans les coques séparées. Microphotographie, prise in vivo par M. FRED VLES, Grossissement 45 fois.

même dans les conditions le plus défavorables, intervenant après le commencement de la ponte.

A côté des ces individus normaux, j'ai rencontré quelques exemplaires de Philine aperta, qui, dans les conditions aussi normales que possible, m'ont donné des pontes, où les coques anormales se trouvaient en abondance. J'ai fait l'étude statistique des coques normales et pluriovulaires dans cinq pontes, pondues dans l'aquarium en même temps que les autres, bien normales, et j'ai obtenu les données suivantes:

I, Sur les 1438 coques il y avait:

756 normales $(52,57 \%)$

664 à deux oufs $(46,18 \%)$

18 à trois oufs $(1,25 \%)$

II. Sur les 1341 coques:

371 normales

$(27,66 \%)$

$826^{\circ}$ à deux reufs $(61,59 \%)$

142 à trois ceufs $(10,60 \%)$

2 à quatre oufs $\quad(0,15 \%)$ 
III. Sur les 1230 coques:

\begin{tabular}{rr}
338 normales & $(31,54 \%)$ \\
810 à deux oufs & $(65,86 \%)$ \\
31 à trois cufs & $(2,52 \%)$ \\
1 à quatre oufs & $(0,08 \%)$ \\
\multicolumn{2}{c}{ IV. Sur les 1600 coques: } \\
314 normales $\quad(19,62 \%)$ \\
841 à deux oufs & $(52,56 \%)$ \\
421 à trois ceufs & $(26,31 \%)$ \\
23 à quatre oufs & $(1,45 \%)$ \\
1 à cinq oufs & $(0,06 \%)$
\end{tabular}

La cinquième ponte renfermait une quantité encore plus surprenante de coques pluriovulaires; c'est pourquoi, en la regardant comme exclusivement précieuse, je n'en ai compté que 181 coques (pour éviter le transport réitéré des œufs sur le porte-objet et le sectionnement $d u$ fil ovigère en fragments isolés). Sur ces 181 coques il y arait:
42 normales
106 à deux oufs
13 à trois œufs
10 à quatre wufs
7 à cinq œufs
2 à six oufs
1 à huit œufs.

En ne comptant que les quatre premières pontes (la einquième étant déjà excessivement anormale), nous royons, que le pourcentage des coques biovulaires peut atteindre le chiffre imposant de $50 \%$ et même plus. En calculant les pourcentages des coques normales et pluriovulaires dans ces quatres pontes ensemble, nous avons:

Sur les 5608 coques (en excluant 1 coque à 5 ceufs):

\begin{tabular}{|c|c|}
\hline 1829 normales & $(32,62 \%)$ \\
\hline 3141 à deux œufs & $(56,00 \%)$ \\
\hline $\begin{array}{l}612 \text { à trois oufs } \\
26 \text { à quatre oufs }\end{array}$ & \\
\hline
\end{tabular}

De ces données il suit, que dans les pontes anormales il y a une stendance* évidente à la formation des coques biovulaires; 
le nombre des coques simples, normales, peut s'abaisser jusqu'à $1 / 5$ da nombre total (ponte IV) et en même temps le pourcentage des coques à trois ceufs s'accroit sensiblement. On dirait, que le nombre des coques triovulaires varie inversement par rapport à celui des coques normales ...

Ajoutons, que H. DE LACAZE-Duthiers n'a signalé, que l'existence de trois oufs au surplus dans la même coque.

Ainsi nous voyons, que chez Philine aperta certains individus (qu'on pourrait comparer aux "parents monstripares", signalés par Chabry chez les Ascidies) - sont capables de fournir des pontes profondement anormales, d'une façon tout à fait spontanée, sans aucune intervention artificielle, tandis que, d'autre part, les individus normaux ne peuvent être forcés par n'importe quel traitement défavorable agissant pendant la ponte et les forçant à déposer plus hâtivement leurs œufs - à produire les coques, renfermant plus d'un seul nuf à la fois. Il n'ya, d'ailleurs, rien d'étonnant de ce que H. De LACAZE-Duthiers fût enduit en erreur d'admettre la possibilité de la production artificielle de cette anomalie: il suffit de tomber, par hasard, sur une série d'individus anormaux ("monstripares«) pour supposer le lien causal entre le traitement des animaux pendant la ponte et le caractère anormal de celle-ci.

La faculté de produire des pontes anormales est donc chez Philine aperta individuelle et spontanée. En soumettant les individus pondeurs des coques anormales - au tracassement mécanique ou électrique, j'ai constaté, que le pourcentage de coques pluriovulaires, pondues dans ces conditions ne s'élève jamais au dessus de celui, qu'on a trouvé dans le lot d'œufs, pondus avant le commencement de l'expérience. Il est done impossible de produire artificiellement les pontes anormales ni même faire augmenter le nombre de coques pluriovulaires dans une ponte déjà primitivement anormale. - Les causes immédiates de l'anomalie nous échappent; ce ne peut être l'inanition des mollusques capturés, car la même anomalie se produit spontanément dans la nature, dans les pontes, déposées sur la grève.

Quand à la question de l'origine et le mode de formation des monstres doubles dans les pontes anormales de Philine - je ne puis pas y apporter encore de faits décisifs, car même dans les pontes sensiblement anormales, les larves doubles ne se rencontrent pas très fréquemment. Je suis porté à admettre, que les vraies poly- 
génèses ne sont pas plus fréquentes chez Philine aperta, que chez n'importe quel autre animal. Dans les quatre pontes anormales, où le pourcentage de coques biorulaires était si élevé $(56 \%)$ - je n'ai trouvé ensuite pas une seale larve double!. Dans les coques à deux ou trois ceufs, dans l'immense majorité des cas (même dans ceux, où le diamètre des coques était sensiblement très petit - les œufs renfermés ensemble évoluaient tout à fait indépendamment l'un de l'autre et à la fin produisaient deux ou trois larves libres. J'avais quelquefois l'occasion de trouver des larves véligères doubles, rassemblant à celles, qui ont été figurées par H. DE LACAzE-Duthiers et M. J. Guiart - dans les pontes cueillies sur la plage (principalement à $\left.S_{A N T E C}\right)$, mais je n'ai jamais pu observer le moment même où leur soudure se produit. Ainsi, en voyant des milliers de coques à deux oufs produire les larves normales - j'étais porté à croire, qu'il y avait ici lieu quelque erreur, et à supposer que l'origine des larves doubles devrait être attribuée à une autre cause; je la cherchais dans les oeufs géants, qui se rencontrent parfois dans les pontes des Philines et j'ai constaté, que de ces oufs se forment des larves normales. J'ai fini tout de même par confirmer la justesse de l'ancienne observation d'H. DE LACAZE-DutHIERs: les monstres doubles naissent ici en réalité par la voie de la soudure secondaire des deux embryons, enfermés dans une coque ovigère commune, seulement cette soudure ne se produit que très rarement, étant donnée la fréquence des coques à deux ovules dans les pontes anormales.

C'est dans ma cinquième ponte anormale (150-ème de toutes celles pondues à l'aquarium, que j'ai étudiées) - qu'à côté d'une grande quantité de coques pluriovulaires, renfermant deux, trois ou même cinq à huit larves normales, indépendantes, - j'ai trouvé dix cas des vrais monstres doubles, appartenant aux types divers de l'union réciproque. Vu l'énorme pourcentage des coques pluriovulaires dans cette ponte, eette quantité tellement insignifiante des larves doubles nous prouve encore une fois, que la présence de deux œufs dans une même coque ne suffit pas encore pour aboutir à leur soudure, ce qui, d'ailleurs, j'ai maintes fois vérifié par les observations directes de toutes les autres pontes anormales. Je supposais d'abord, que la soudure des deux larves se produit dans des coques exceptionellement étroites et pour le vérifier j'entrepris une longue série des mensurations comparatives des diamètres des coques normales et pluriovulaires, mais je n'ai pas pu trouver de différences appréciables entre les dimensions des coques renfermant deux larves 
véligères indépendantes et celles aux monstres doubles. D'ailleurs il suffit d'examiner notre fig. 2 pour ce convaincre, que même les larves disposant d'une coque suffisamment spacieuse pour qu'elles y puissent se mouvoir librement sans être forcées à contracter la sou-

Fig. 2.

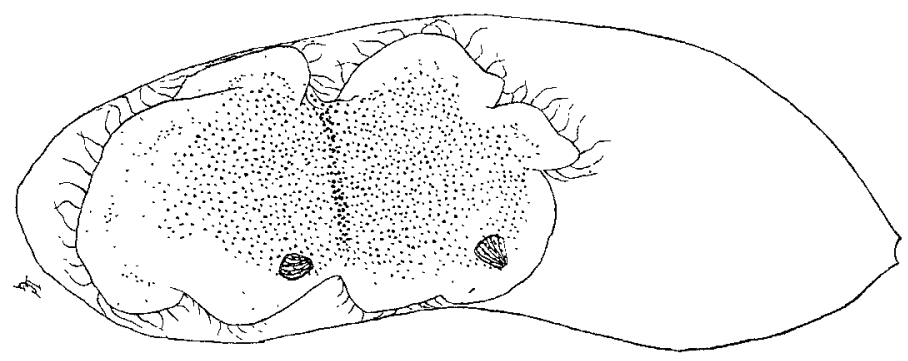

Un monstre donble Iarvaire de Philine aperitu daıs une coque ovigère aux grandes dimensions. Chambre elaire. Grossissement 250 fois, Dessin de M. I. Garliera.

dure réciproque - peuvent néanmoins aboutir à la formation d'un monstre double. La question devient encore plus compliquée quand nous avons à faire aux cas - non signalés ni par H. DE LACAZEDuthiers ni par M. Guiart - où dans une coque à trois ceufs, deux d'entre eux se soudent en formant un monstre double, tandis que le troisième reste libre et érolue en une larve normale. Dans la même ponte (cinquième) j'ai trouvé un cas tout à fait exceptionnel, représenté sur la fig. 3 , où des six ceufs, enfermés dans une

Fig. 3.

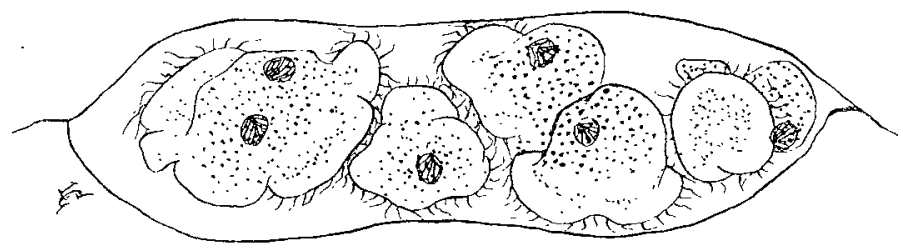

Coque ovigère anormale de Philine aperta, contenant un monstre double (à gauche) et quatre larves normules. Chambre claire. Grossissement 125 fois. Dessin de M. L. GarreTA.

coque commune - quatre ont donné des larres normales, tandis qu'à côté de celles-ci deux autres germes se sont soudés en un monstre double typique.

Quel est alors le moment décisif de cette soudure? L'étroitesse de la coque n'est pas à invoquer évidemment: dans une autre coque, 
beaucoup plus étroite, j'ai vu huit oeufs donner autant de larves indépendantes. Leur mouvements étaient très gênés, elles se serraient très étroitement les unes contre les autres, en produisant parfois l'impression de la soudure, ce qui ensuite s'est montré illusoire, et, enfin, elles abandonnaient leur coque séparement. Alors est-ce le rapprochement accidentel par des parties similaires, le plus aptes à la fusion réciproque? Dans le matériel, que j'ai étudié, à part des monstres doubles strictement symmétriques, liés par les parties homologues, - j'ai constaté la présence des larves soudées par les regions tout à fait dissemblables: p. ex. la partie aborale d'un embryon peut se souder à la region ventrale ou même dorsale d'un autre.... Il existe aussi des soudures tout à fait superficielles, réduites á une région très limitée de revêtement ectodermique commun. Ainsi le type de monstruosité, représenté sur la fig. $7 \mathrm{du}$ mémoire d'H. DE LaCAzE-Duthiers doit être considéré comme l'exemple de ces soudures superficielles, qui, d'ailleurs, peuvent n'être que transitoires: les larves, unies par la face de l'ébauche de leur pied, restent dans cette position quelque temps après leur sortie de la coque, mais se séparent ensuite assez facilement.

Je n'insisterai pas ici sur la description des diverses formes des larves doubles, qui ont été déjà figurées par les deux auteurs cités, j'ajouterai seulement que la période où s'accomplit la soudure - contrairement à ce qu'on pourrait admettre à priori - doit être cherchée dans les stades qui suivent la gastrulation et l'achevement de la forme cordiforme de la larve. Il paraît, que cette soudure pent se produire même entre les larves mobiles, pourvues déjà de leurs disques moteurs.

A côté des coques pluriovulaires et des monstres doubles, on rencontre parfois dans les pontes des Philines - quoique très rarement - une autre anomalie non moins intéressante. J'ai déjà dit, qu'en exerçant une traction sur le filament ovigère, sortant de l'orifice génital de l'animal, on arrive quelquefois à faire sortir les enveloppes coquillières très étroites, renfermant plus d'un cuf. Or il paraît, que cette faculté de produire de pareilles coques tubiformes n'est qu'aussi individuelle, que celle de pondre les larges coques pluriovulaires. Deux on trois fois j'ai vu de telles coques anormales se produire spontanément, dans les conditions normales; les ceufs qui y étaient enfermés, subissaient toujours une pression visiblement assez forte de la part des parois de la coque, mais parfois ils réussissaient à se développer jusqu'au stade des larves pour- 
vues de cils vibratils. Dans ces conditions la soudure des germes s'opérait de très bonne heure, mais elle n'aboutissait jamais à la formation de larves doubles normalement conformées: il se produit ici une masse commune d'espèce d'une larve géante, dont il est impossible de discerner in vivo la disposition des organes internes. Les "yeux anaux" (ébauches des reins) sont ici disseminés sans ordre apparant dans la masse difforme et c'est seulement d'après le nombre de ces organes, d'un rouge vif, qu'on peut reconnaître le nombre primitif d'individus, qui se sont soudés ${ }^{1}$ ). En observant de telles larves, composées de trois ou quatre germes, renfermés dans une coque étroite, tubiforme, aux bouts éffilés, j'ai vu, que leurs disques rotateurs ne se déreloppent pas (faute de place, probablement), mais les cils vibratils se forment tout de même, en donnant à une telle larve géante l'aspect d'un Turbellaire. Il est inutile d'ajouter, que la durée de la vie de telles larves est très limitée, et qu'elles ne parviennent jamais à abandonner leurs coques.

Enfin je signalerai une anomalie singulière, qui a été dessinée, mais non décrite par H. DE LACAZE-Duthiers: e'est la présence des coques vides, dépourvues d'œufs (1. cit. Pl. XV une petite coque vide entre les fig. 6 et 7), mêlées aux coques, contenant les embryons: Cette anomalie ne se rencontre guère (d'après ce que j'ai observé) dans les pontes cueillies sur la plage, mais, au contraire, elle est assez fréquente dans celles pondues par les animanx qui ont été capturés depuis quelque semaines. Vers la sixième semaine de captivité mes Philines m'ont donné plusieurs fois l'occasion d'étudier les coques vides d'aspect et de diamètres très variés. Les dimensions de ces coques peurent être sensiblement inférieures à celles des coques embryonnées (c'est bien le cas représenté par H. DE LACAZEDuthiers), mais aussi elles penvent être égales aux coques ordinaires ou même les surpasser en diamètre et atteindre les dimensions des coques pluriovulaires, même des celles à cinq ou six embryons. Parfois - et cela arrive dans les pontes contenant une quantité d'oeufs inférieure à la normale - j'ai vu des amas considérables de coques vides, intercalés entre les coques normales peu nombrenses. Il me semble, que l'apparition de ces coques vides est liée à un certain degré d'ẹpuisement de l'animal capturé et mal nourri et indique

1) Je ne crois pas qu'on pourrait - sans grande réserve - comparer ces larves géantes à celles, décrites par Metschnikoff, Sala, zur Strrassen, Driesch et MoRgan. 
un particulier manque de corrélation physiologique entre le fonctionnement de l'appareil coquillier et la productivité de la glande génitale.

\section{Résumé.}

1) Chez Philine aperta les pontes anormales, contenant les coques pluriovulaires ne peuvent pas être provoquées artificiellement par le tracassement de l'animal pendant la ponte. Les individus normaux ne déposent jamais - même dans les conditions le plus défarorables, agissant après le commencement de la poute - que des cufs normanx.

2) Les pontes pluriovulaires sont toujours produites d'une façon spontanée par des individus »monstripares«.

3) Il est impossible de faire augmenter par la voie artificielle - le nombre des coques pluriovulaires, pondues par les individus anormaux.

4) Dans les pontes anormales le pourcentage de coques à deux oeufs (le plus fréquentes) peut dépasser $50 \%$; celui de coques à trois ceufs peut atteindre $10 \%$. Il existe de coques à quatre et jusqu'à huit ceufs, enfermés ensemble.

5) Les ceufs, contenus dans les coques pluriovulaires, le plus souvent se développent en larves indépendantes, normales, et ne donnent qu'assez rarement de monstres doubles, dont la fréquence ordinairement n'est pas plus grande chez Philine aperta que chez d'autres animaux. Les larves doubles se forment, comme l'a déjà demontré H. DE LACAZE-DUTHIERs, par la soudure secondaire des germes primitivement indépendants. Cette soudure ne se fait pas nécessairement par les régions homologues. Les dimensions restreintes des coques pluriovulaires ne sauraient être invoquées comme cause de la soudure des embryons.

6) Dans les coques pluriovulaires trop étroites et tubiformes, les cufs se soudent en une larve géante, difforme, pourvue de cils, où on ne peut discerner le nombre d'individus primitifs que par celui d'ébauches des reins.

7) Les coques vides, désembryonnées, se produisent dans les pontes des animaux épuisés par une captivité prolongée.

Varsovie, Laboratoire Zootomique de l'Université,

Février 1910. 


\section{Zusammenfassung,}

1) Bei Philine aperta lassen sich die abnormen Gelege, welche die Schalen mit mehreren Eiern enthalten, nicht künstlich durch die Beunruhigung des Tieres während der Eiablage hervorrufen. Die normalen Individuen legen niemals, - selbst nicht unter den ungüntigsten Bedingungen, die nach dem Beginn der Eiablage einwirken, - andre als normale Eier.

2) Die Gelege mit mehreren Eiern in einer Schale werden immer in spontaner Weise von besonderen smonstriparen Individuen hervorgebracht.'

3) Es ist nicht müglich, auf künstlichem Wege die Anzahl der mehrere Eier enthaltenden Schalen zu vermehren, welche von abnormen Individuen gelegt werden.

4) In den abnormen Eiern überschreitet der Prozentsatz der Schalen mit zwei Eiern (diese sind am hänfigsten) $50 \%$; derjenige der Schalen mit drei Eiern kann 10\% erreichen. Es kommen Schalen mit vier bis zu acht Eiern vor, die alle zusammen eingeschlossen sind.

5) Die in den mehreirigen Schalen eingeschlossenen Eier entwickeln sich in den meisten Fällen zu unabhängigen normalen Larven und ergeben nur recht selten Doppelmißbildungen, deren Vorkommen bei Philine aperta gewöhnlich nicht häufiger ist, als bei andern Tieren. Die Doppellarven bilden sich, wie dies bereits LACAZE-DUTHLER gezeigt hat, sekundür durch Vereinigung der primär voneinander unabhängigen Keime. Bei der Vereinigung legen sich nicht notwendig homologe Bezirke zusammen. Die beschränkten Dimensionen der mehrere Eier enthaltenden Schalen lassen sich nicht als Ursache der Verschmelzung der Embryonen verantwortlich machen.

6) In den Schalen mit mehreren Eiern, welche zu eng und röhrenförmig sind, verschmelzen die Eier zu einer Riesenlarve, die unförmig und mit Wimpern versehen ist, und in welcher man die Anzahl der verschmolzenen Primärindividuen nur an.der Zahl der Nierenanlagen erkennen kann.

7) Die leeren Schalen ohne Embryonen treten in den Gelegen von Tieren auf, welche durch Iange Gefangenschaft heruntergekommen sind.

(Übersetzt von W. Gebhardt, den 2. März 1910.) 